\title{
IAMJ
}

INTERNATIONAL

AYURVEDIC

MEDICAL JOURNAL

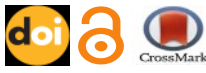

Research Article

ISSN: 23205091

Impact Factor: 5.344

\section{A COMPARATIVE PHARMACEUTICO ANALYTICAL STUDY OF BHAGANDHARA- HARA LEPA WITH ITS MODIFIED FORM AS A HYDROGEL}

\author{
Hari Anand Tripathi ${ }^{1}$, J. Dinesh Nayak $^{2}$, Sathyanarayana $B^{3}$ \\ ${ }^{1}$ PG. Scholar Dept. Of Rasashastra \& Bhaishajya Kalpana, M.I.A.M.S, Manipal, Karnataka, India \\ ${ }^{2} \mathrm{MD}$ (Ayu), Professor, PG Dept. of Rasashastra and Bhaishajya Kalpana, \\ Muniyal Institute of Ayurveda Medical Sciences, Manipal, Karnataka, India \\ ${ }^{3}$ M.D. (Ayu), Principal and H.O.D., Dept. of Rasashastra and Bhaishajya Kalpana \\ Muniyal Institute of Ayurveda Medical Sciences, Manipal, Karnataka, India
}

Email: dr.harianandtripathi@gmail.com

\section{https://doi.org/10.46607/iamjp04042020}

(Published online: May 2020)

Open Access

(C) International Ayurvedic Medical Journal, India 2020

Article Received: 09/04/2020 - Peer Reviewed: 20/04/2020 - Accepted for Publication: 04/05/2020

Check for updates

\section{ABSTRACT}

Objective of the study: To the develop standard operative procedures of Bhagandharahara lepa and change the form of Bhagandharahara lepa into the form of the Hydrogel. To comparatively analysis Bhagandharahara lepa and its pharmaceutically modified form of Hydrogel with classical and advanced analytical techniques. Methods: Pharmaceutical study: Bhagandharahara lepa and its modified form as a Hydrogel is prepared with standard household parameters. Analytical study: Comparative analysis of both Bhagandharahara lepa and its modified form as a Hydrogel will be comparatively analysed with suitable physicochemical parameters and advanced instrumental methods of analysis. Results: In Stability evaluation of BHL, classically prepared lepa got decomposed next day with foul smell and become darker mustard field in colour, so lepa (wet form) stability remains within 24 hrs. and here physical and chemical stability of dried form of lepa churna is good throughout 3 months of period and in Stability evaluation of BHG prepared hydrogel Light Ochre in colour with easily spreading and physicochemical parameters also like homogeneity and extrudability, viscosity, $\mathrm{pH}$, irritancy test, melting point within limit there is no any major changes throughout the stability period 3 months. So Bhagandharahara Hydrogel is almost remained 
stable till 3 months. Conclusions: As lepa is having shorter shelf life, it cannot be preserved to longer time, so in this study its modification to Hydrogel form facilitates a longer shelf life. Due to some special features of Hydrogel like extrudability, spreadability, viscosity, $\mathrm{pH}$, homogeneity it is easy to apply to the target site.

Keywords: Bhagandhara, Lepa Kalpana, Hydrogel.

\section{INTRODUCTION}

Ayurveda with its specified branch of Shalya Tantra has found success in treating the Bhagandhara with various treatment like application of kshara, varti, taila etc. $^{1}$

The present study of Bhagandharahara lepa ${ }^{2,3}$ is selected. As application of lepa in the cases of Bhagandhara many a times may not be convenient especially in case of long track fistulae or multiple track fistulae, an effort is planned to modify the lepa into a Hydrogel form to make it easier and convenient for the surgeon to apply the gel into the communicating tract of fistula in ano with ease.

Herbal drugs were not used precisely in modern pharmacy due to their lengthy process and various procedure, recently a trend of modifying such herbal formulation to pharmaceutically modified form such as cream, gel, ointment etc. have been started.

These pharmaceutically modified forms of herbal formulations have increased their market value and ease in application also enhanced their therapeutic efficacy, shelf life and acceptability. Considering the above fact, the present study is framed.

\section{Aim and Objectives}

1. To develop a standard method of preparation of Bhagandharahara lepa.

2. To convert the Bhagandharhara lepa into a Hydrogel form.

3. To comparatively analyse both Bhagandharhara lepa and its pharmaceutically modified form of a Hydrogel with classical and advanced analytical techniques.

\section{Materials and Method}

The reference of Bhagandharahara lepa is from $\mathrm{Sa}$ hasrayogam. It is poly herbal formulation in the form of lepa, here Haridra, Haritaki, Nimba patra, Arka moola, Saindhava lavana and Takra 6 ingredients are the described. All the 5 drugs (Haridra ${ }^{4}$, Haritaki $^{5}$,
Nimbapatra $^{6}$, Saindhava lavana ${ }^{7}$, Arkaroola $^{8}$ ) 1 part each and takra 10 parts (q.s) were used for lepa preparation.

\section{Method of preparation of lepa}

All the drugs mentioned in classical reference is collected. The drugs are individually powdered and sieved to get a micro fine powder and mardana is done with Takra and lepa is obtained. This prepared lepa is divided into two parts, one part of lepa is stored in a glass air tight container and another part of prepared lepa is placed in a steel tray for dry procedure with the help of vacuum oven drier finally dried form of lepa is obtained and this dried form of lepa is collected from steel tray and triturated well with the help of Mortar and pestle finally lepa churna is obtained micro fine form and stored in a glass air tight container.

[Here as we know wet form of lepa is having short shelf life, this prepared lepa were divided into 2 parts for further subjected analysis purpose.]

\section{Method of preparation of Hydrogel}

It consists of 3 steps.

Step 1- Preparation of Lepa churna (To dried form)

Step 2-Preparation of Drug extract water

Step 3- Preparation of Hydrogel ${ }^{10}$

STEP 1: Preparation of Bhagandharahara Lepa churna

All the drug mentioned in the preparation of bhagandharahara lepa are individually powdered and mixed well with buttermilk \& vacuum dried.

\section{STEP 2: Preparation of Drug extract water}

The dried form of Bhagandharahara lepa churna is macerated with the distilled water in the ratio of $1: 10$ in a closed conical flask for twenty-four hours, shaking frequently during six hours and kept stagnant for eighteen hours. After eighteen hours the liquid is filtered using the filter paper and drug extract water is obtained. 


\section{STEP 3: Preparation of Hydrogel}

Quantity sufficient Drug extract water is taken in a beaker and at the same time, weighed quantity of Sodium benzoate ${ }^{11} 0.5 \%\left(\mathrm{C}_{7} \mathrm{H}_{5} \mathrm{O}_{2} \mathrm{Na}\right)$ is added and mixed with drug extract water.

Weighed quantity of Carbomer ${ }^{12} 940 \quad 5 \% \quad$ w/v is dissolved with drug extract mixture \& kept for 24 hours.

After 24 hours weighed quantity of PEG $4002 \%$ (Polyethylene glycol $^{13}$ ) was taken in another beaker

\section{Results}

\section{Raw Material Standardization:}

Table 1: Showing Physico-chemical evaluation of raw materials

\begin{tabular}{|l|l|l|l|l|l|l|}
\hline Drug name & Total ash & $\begin{array}{l}\text { Water } \\
\text { extractive }\end{array}$ & $\begin{array}{l}\text { Alcohol soluble } \\
\text { Extractive }\end{array}$ & $\begin{array}{l}\text { Acid insoluble } \\
\text { ash }\end{array}$ & Loss on drying & pH \\
\hline Nisa (Haridra) & $6.95 \%$ & $12.96 \%$ & $9.20 \%$ & $0.95 \%$ & $6.82 \%$ \\
\hline Pathya (Haritaki) & $4.05 \%$ & $82.64 \%$ & $41.74 \%$ & $0.70 \%$ & $4.14 \%$ & - \\
\hline Nimba patra & $9.95 \%$ & $27.00 \%$ & $25.36 \%$ & $3.90 \%$ & $4.98 \%$ & - \\
\hline Arka moola & $3.60 \%$ & $7.84 \%$ & $4.56 \%$ & $1.00 \%$ & $10.34 \%$ \\
\hline Saindhava lavana & $0.5 \%$ & - & - & $1 \%$ & $0.1 \%$ & - \\
\hline Takra & - & - & - & - & 8.90 \\
\hline
\end{tabular}

Table 2: Showing Phytochemical evaluation of raw materials in water extract and methanol extract

\begin{tabular}{|c|c|c|c|c|c|c|c|c|c|c|c|c|c|c|c|c|c|c|}
\hline \multirow[t]{2}{*}{$\begin{array}{l}\text { Drug } \\
\text { name }\end{array}$} & \multicolumn{2}{|c|}{ Alkaloids } & \multicolumn{2}{|c|}{ Tannins } & \multicolumn{2}{|c|}{$\begin{array}{l}\text { Amino } \\
\text { acids }\end{array}$} & \multicolumn{2}{|c|}{ Sugars } & \multicolumn{2}{|c|}{$\begin{array}{l}\text { Glycosid } \\
\text { es }\end{array}$} & \multicolumn{2}{|c|}{$\begin{array}{l}\text { Phenolic } \\
\text { compound }\end{array}$} & \multicolumn{2}{|c|}{$\begin{array}{l}\text { Flavonoid } \\
\text { S }\end{array}$} & \multicolumn{2}{|c|}{ Proteins } & \multicolumn{2}{|c|}{ Saponins } \\
\hline & $\begin{array}{l}\text { Wat } \\
\text { er } \\
\text { extr } \\
\text { act }\end{array}$ & $\begin{array}{l}\text { met } \\
\text { han } \\
\text { ol } \\
\text { extr } \\
\text { act }\end{array}$ & $\begin{array}{l}\text { wa } \\
\text { ter } \\
\text { ex } \\
\text { tra } \\
\text { ct }\end{array}$ & $\begin{array}{l}\text { met } \\
\text { han } \\
\text { ol } \\
\text { extr } \\
\text { act }\end{array}$ & $\begin{array}{l}\text { wa } \\
\text { ter } \\
\text { ex } \\
\text { tra } \\
\text { ct }\end{array}$ & $\begin{array}{l}\text { met } \\
\text { han } \\
\text { ol } \\
\text { extr } \\
\text { act }\end{array}$ & $\begin{array}{l}\text { wa } \\
\text { ter } \\
\text { ex } \\
\text { tra } \\
\text { ct }\end{array}$ & $\begin{array}{l}\text { met } \\
\text { ha- } \\
\text { nol } \\
\text { ex- } \\
\text { tract }\end{array}$ & $\begin{array}{l}\text { wa } \\
\text { ter } \\
\text { ext } \\
\text { rac } \\
\mathrm{t}\end{array}$ & $\begin{array}{l}\text { met } \\
\text { han } \\
\text { ol } \\
\text { ext } \\
\text { ract }\end{array}$ & $\begin{array}{l}\text { wat } \\
\text { er } \\
\text { extr } \\
\text { act }\end{array}$ & $\begin{array}{l}\text { met } \\
\text { han } \\
\text { ol } \\
\text { extr } \\
\text { act }\end{array}$ & $\begin{array}{l}\text { wa } \\
\text { ter } \\
\text { ext } \\
\text { rac } \\
\text { t }\end{array}$ & $\begin{array}{l}\text { met } \\
\text { han } \\
\text { ol } \\
\text { extr } \\
\text { act }\end{array}$ & $\begin{array}{l}\text { wa } \\
\text { ter } \\
\text { ext } \\
\text { rac } \\
\mathrm{t}\end{array}$ & $\begin{array}{l}\text { met } \\
\text { han } \\
\text { ol } \\
\text { extr } \\
\text { act }\end{array}$ & $\begin{array}{l}\text { wa } \\
\text { ter } \\
\text { ext } \\
\text { rac } \\
\text { t }\end{array}$ & $\begin{array}{l}\text { meth } \\
\text { anol } \\
\text { extra } \\
\text { ct }\end{array}$ \\
\hline $\begin{array}{l}\text { Nisa } \\
\text { (Harid) }\end{array}$ & + & + & + & + & - & - & + & + & + & + & - & + & - & + & + & + & - & - \\
\hline $\begin{array}{l}\text { Pathya } \\
\text { (Haritak } \\
\text { ) }\end{array}$ & - & - & + & + & - & - & + & + & + & + & + & + & - & - & + & + & - & - \\
\hline $\begin{array}{l}\text { Nimba } \\
\text { patra }\end{array}$ & - & - & + & + & - & - & + & - & + & - & + & + & - & - & + & + & + & + \\
\hline $\begin{array}{l}\text { Arka } \\
\text { moola }\end{array}$ & - & - & - & - & - & - & + & + & + & + & - & - & - & - & - & - & + & - \\
\hline
\end{tabular}

2. Finished Product Standardization: (A) Bhagandharahara Lepa:

Table 3: Organoleptic evaluation of Bhagandharahara lepa (wet form)

\begin{tabular}{|l|l|l|l|l|l|}
\hline BATCH & Time (day) & Evaluation parameters & & \\
\cline { 3 - 5 } & & Colour & Odour & Texture & Consistency \\
\hline \multirow{2}{*}{ BHL } & 0 & Mustard field & Smell of Nimba & Smooth & Paste \\
\cline { 2 - 5 } & 1 & Dark Mustard field & Foul Smell & Smooth & Paste \\
\hline
\end{tabular}


Table 4: Showing Organoleptic evaluation of Bhagandharahara lepa churna (dried form)

\begin{tabular}{|l|l|l|l|l|l|}
\hline \multirow{2}{*}{ BATCH } & \multirow{2}{*}{ Time (day) } & Evaluation parameters & & \\
\cline { 3 - 6 } & & Colour & Odour & Texture & Consistency \\
\hline \multirow{3}{*}{ BHL } & 0 & Mustard field & Smell of Nimba & Smooth & Fine powder \\
\hline & 15 & Mustard field & Smell of $\mathrm{Nimba}$ & Smooth & Fine powder \\
\hline & 30 & Mustard field & Smell of $\mathrm{Nimba}$ & Smooth & Fine powder \\
\hline & 45 & Mustard field & Smell of Nimba & Smooth & Fine powder \\
\hline & 60 & Mustard field & Smell of Nimba & Smooth & Fine powder \\
\hline & 75 & Mustard field & Smell of Nimba & Smooth & Fine powder \\
\hline & 90 & Mustard field & Smell of Nimba & Smooth & Fine powder \\
\hline
\end{tabular}

Table 5: Showing the table physico-chemical evaluation of Bhagandharahara Lepa (wet form)

\begin{tabular}{|l|l|l|}
\hline Parameter & BHL & 1 \\
\hline Time (Day) & 0 & 4.85 \\
\hline $\mathrm{P}^{\mathrm{H}}(10 \%$ aqueous extract) & 4.68 & \\
\hline
\end{tabular}

Table 6: Showing Physico-chemical evaluation of Bhagandharahara lepa churna (dried form)

\begin{tabular}{|l|l|l|l|l|l|l|l|}
\hline Parameter & BHL & \multicolumn{7}{l|}{$l$} \\
\hline Time (Day) & 0 & 15 & 30 & 45 & 60 & 75 & 90 \\
\hline Loss on drying (\%) & 4.7 & 4.58 & 4.67 & 4.91 & 4.85 & 4.88 & 4.83 \\
\hline Total ash (\%) & 17.35 & 17.49 & 16.75 & 17.70 & 17.25 & 16.85 & 17.05 \\
\hline Acid insoluble ash (\%) & 2.14 & 2.06 & 2.18 & 2.06 & 2.06 & 2.04 & 2.26 \\
\hline Alcohol-soluble extractive (\%) & 29.00 & 28.90 & 30.05 & 29.88 & 29.58 & 29.06 & 29.33 \\
\hline Water soluble extractive (\%) & 64.88 & 65.4 & 64.70 & 64.59 & 64.80 & 64.58 & 64.70 \\
\hline $\mathrm{P}^{\mathrm{H}}$ (10\% aqueous extract) & 4.62 & 4.64 & 4.65 & 4.60 & 4.68 & 4.50 & 4.83 \\
\hline Particle size & $\begin{array}{l}\text { Micro } \\
\text { fine }\end{array}$ & Micro fine & $\begin{array}{l}\text { Micro } \\
\text { fine }\end{array}$ & Micro fine & Micro fine & $\begin{array}{l}\text { Micro } \\
\text { fine }\end{array}$ & $\begin{array}{l}\text { Micro } \\
\text { fine }\end{array}$ \\
\hline
\end{tabular}

Table 7: Showing Phyto chemical evaluation of Bhagandharahara lepa churna in water soluble extractive and Methanol soluble Extractive

\begin{tabular}{|c|c|c|c|c|c|c|c|c|c|c|c|c|c|c|}
\hline Parameter & \multicolumn{7}{|c|}{ BHL water soluble extractive } & \multicolumn{7}{|c|}{ BHL Methanol soluble Extractive } \\
\hline Time (Day) & 0 & 15 & 30 & 45 & 60 & 75 & 90 & 0 & 15 & 30 & 45 & 60 & 75 & 90 \\
\hline Alkaloids (Mayer's test) & -ve & -ve & -ve & -ve & -ve & -ve & -ve & + & + & + & + & + & + & + \\
\hline Sugars (Fehling's solution test) & + & + & + & + & + & + & + & + & + & + & + & + & + & + \\
\hline Glycosides (Keller kiliyani test) & + & + & + & + & + & + & + & + & + & + & + & + & + & + \\
\hline Phenolic compound & + & + & + & + & + & + & + & + & + & + & + & + & + & + \\
\hline Flavonoids (Shinoda test) & -ve & -ve & -ve & -ve & -ve & -ve & -ve & -ve & -ve & -ve & -ve & -ve & -ve & -ve \\
\hline Nin-Hydrin test (Amino acids) & -ve & -ve & -ve & -ve & -ve & -ve & -ve & -ve & -ve & -ve & -ve & -ve & -ve & -ve \\
\hline proteins (Biuret test) & -ve & -ve & -ve & -ve & -ve & -ve & -ve & + & + & + & + & + & + & + \\
\hline Tannins (Ferric chloride test) & + & + & + & + & + & + & + & + & + & + & + & + & + & + \\
\hline Saponins & -ve & -ve & -ve & -ve & -ve & -ve & -ve & -ve & -ve & -ve & -ve & -ve & -ve & $-\mathrm{ve}$ \\
\hline
\end{tabular}

\section{HPTLC Determination}

1) Standard curcumin: Rf 0.24 AUC: 40490.1

2) BHL: Rf 0.26 AUC: 13127.8 
Table 8: Showing HPTLC determination of Bhagandharahara lepa churna Quantification of Curcumin in sample

\begin{tabular}{|l|l|}
\hline Sample & Percentage content of Curcumin \\
\hline BHL & $6.28 \%$ \\
\hline
\end{tabular}

Stability Test: (Physical and Chemical Stability)

The stability study was performed as per ICH guidelines and formulation were stored under room temperature stability conditions (Temperature $25^{\circ} \mathrm{C} \pm 2^{\circ} \mathrm{C}$,
Humidity $\quad 65 \% \pm 5 \% \mathrm{RH}$ ) were carried for a period of 3 months. The physical and chemical analysis was assessed periodically at 15 days interval for a period of 3 months.

Table 9: Showing Physico Organoleptic Stability evaluation of Bhagandharahara lepa (wet form)

\begin{tabular}{|c|c|c|c|c|c|}
\hline \multirow[t]{2}{*}{ BATCH } & \multirow{2}{*}{$\begin{array}{l}\text { Time } \\
\text { (day) }\end{array}$} & \multicolumn{4}{|c|}{ Evaluation parameters } \\
\hline & & Colour & Odour & Texture & Consistency \\
\hline \multirow[t]{2}{*}{ BHL } & 0 & Mustard field & Smell of Nimba & Smooth & Paste \\
\hline & 1 & Dark Mustard field & Foul Smell & Smooth & Paste \\
\hline
\end{tabular}

Table 10: Showing Physico-chemical stability evaluation of Bhagandharahara Lepa (wet form)

\begin{tabular}{|l|l|l|}
\hline Parameter & BHL & \\
\hline Time (Day) & 0 & 1 \\
\hline $\mathrm{P}^{\mathrm{H}}(10 \%$ aqueous extract) & 4.68 & 4.85 \\
\hline
\end{tabular}

Table 11: Showing Physico-organoleptic Stability evaluation of Bhagandharahara lepa churna (dried form)

\begin{tabular}{|l|l|l|l|l|l|}
\hline \multirow{2}{*}{ BATCH } & $\begin{array}{l}\text { Time } \\
\text { (day) }\end{array}$ & Evaluation parameters & Toxture & Consistency \\
\hline \multirow{2}{*}{ BHL } & 0 & Mustard field & Smell of $\mathrm{Nimba}$ & Smooth & Fine powder \\
\hline & 15 & Mustard field & Smell of $\mathrm{Nimba}$ & Smooth & Fine powder \\
\hline & 30 & Mustard field & Smell of $\mathrm{Nimba}$ & Smooth & Fine powder \\
\hline & 45 & Mustard field & Smell of $\mathrm{Nimba}$ & Smooth & Fine powder \\
\hline & 60 & Mustard field & Smell of $\mathrm{Nimba}$ & Smooth & Fine powder \\
\hline & 75 & Mustard field & Smell of $\mathrm{Nimba}$ & Smooth & Fine powder \\
\hline & 90 & Mustard field & Smell of $\mathrm{Nimba}$ & Smooth & Fine powder \\
\hline
\end{tabular}

Table 12: Showing Physico-chemical Stability evaluation of Bhagandharahara lepa churna (dried form)

\begin{tabular}{|l|l|l|l|l|l|l|l|}
\hline Parameter & BHL & \multicolumn{5}{l|}{$l \mid$} \\
\hline Time (Day) & 0 & 15 & 30 & 45 & 60 & 75 & 90 \\
\hline Loss on drying (\%) & 4.7 & 4.58 & 4.67 & 4.91 & 4.85 & 4.88 & 4.83 \\
\hline Total ash (\%) & 17.35 & 17.39 & 18.16 & 17.88 & 17.41 & 18.18 & 18.11 \\
\hline Acid insoluble ash (\%) & 2.04 & 2.06 & 2.18 & 2.14 & 2.56 & 2.04 & 2.26 \\
\hline Alcohol-soluble extractive (\%) & 29 & 28.90 & 30.05 & 29.88 & 29.58 & 29.06 & 29.33 \\
\hline Water soluble extractive (\%) & 64.88 & 65.4 & 64.70 & 64.59 & 64.80 & 64.58 & 64.70 \\
\hline pH (10\% aqueous extract) & 4.62 & 4.64 & 4.65 & 4.60 & 4.68 & 4.50 & 4.83 \\
\hline
\end{tabular}




\section{Bhagandharahara Hydrogel:}

Table 13: Showing Organoleptic evaluation of Bhagandharahara Hydrogel

\begin{tabular}{|c|c|c|c|c|c|c|}
\hline & Time (Day) & Colour & Odour & Texture & Consistency & Spreadability \\
\hline \multirow[t]{7}{*}{ BHG } & 0 & $\begin{array}{l}\text { Light Ochre (colour } \\
\text { code 7926) }\end{array}$ & $\begin{array}{l}\text { Characteristics pungent } \\
\text { odour }\end{array}$ & Smooth & $\begin{array}{l}\text { Porosity and } \\
\text { soft }\end{array}$ & $\begin{array}{l}\text { Easily spread- } \\
\text { ing }\end{array}$ \\
\hline & 15 & brownish yellow & $\begin{array}{l}\text { Characteristics pungent } \\
\text { odour }\end{array}$ & Smooth & $\begin{array}{l}\text { Porosity and } \\
\text { soft }\end{array}$ & $\begin{array}{l}\text { Easily spread- } \\
\text { ing }\end{array}$ \\
\hline & 30 & brownish yellow & $\begin{array}{l}\text { Characteristics pungent } \\
\text { odour }\end{array}$ & Smooth & $\begin{array}{l}\text { Porosity and } \\
\text { soft }\end{array}$ & $\begin{array}{l}\text { Easily spread- } \\
\text { ing }\end{array}$ \\
\hline & 45 & brownish yellow & $\begin{array}{l}\text { Characteristics pungent } \\
\text { odour }\end{array}$ & Smooth & $\begin{array}{l}\text { Porosity and } \\
\text { soft }\end{array}$ & $\begin{array}{l}\text { Easily spread- } \\
\text { ing }\end{array}$ \\
\hline & 60 & brownish yellow & $\begin{array}{l}\text { Characteristics pungent } \\
\text { odour }\end{array}$ & Smooth & $\begin{array}{l}\text { Porosity and } \\
\text { soft }\end{array}$ & $\begin{array}{l}\text { Easily spread- } \\
\text { ing }\end{array}$ \\
\hline & 75 & brownish yellow & $\begin{array}{l}\text { Characteristics pungent } \\
\text { odour }\end{array}$ & Smooth & $\begin{array}{l}\text { Porosity and } \\
\text { soft }\end{array}$ & $\begin{array}{l}\text { Easily spread- } \\
\text { ing }\end{array}$ \\
\hline & 90 & brownish yellow & $\begin{array}{l}\text { Characteristics pungent } \\
\text { odour }\end{array}$ & Smooth & $\begin{array}{l}\text { Porosity and } \\
\text { soft }\end{array}$ & $\begin{array}{l}\text { Easily spread- } \\
\text { ing }\end{array}$ \\
\hline
\end{tabular}

Table 14: Showing Homogeneity evaluation of Bhagandharahara Hydrogel

\begin{tabular}{|l|l|l|l|l|l|l|l|}
\hline Parameter & BHG \\
\hline Time (Day) & 0 & 15 & 30 & 45 & 60 & 75 & 90 \\
\hline Homogeneity test & ++ & + & + & + & + & + & + \\
\hline
\end{tabular}

$++=$ very good, $+=$ good

Table 15: Showing the $\mathrm{pH}$ evaluation of Bhagandharahara Hydrogel.

\begin{tabular}{|l|l|l|l|l|l|l|l|}
\hline Parameter & BHG & \multicolumn{7}{l|}{$l \mid$} \\
\hline Time (Day) & 0 & 15 & 30 & 45 & 60 & 75 & 90 \\
\hline $\mathrm{P}^{\mathrm{H}}(10 \%$ aqueous extract & 4.90 & 4.63 & 4.46 & 4.41 & 4.41 & 4.29 & 4.29 \\
\hline
\end{tabular}

Table 16: Showing Viscosity evaluation of Bhagandharahara Hydrogel

\begin{tabular}{|l|l|l|l|l|l|l|l|}
\hline Parameter & BHG & \multicolumn{1}{l|}{} \\
\hline Time (Day) & 0 & 15 & 30 & 45 & 60 & 75 & 90 \\
\hline Viscosity (cp) & $>30,650$ & $>30,650$ & $>30,650$ & $>30,650$ & $>30,650$ & $>30,650$ & $>30,650$ \\
\hline
\end{tabular}

Table 17: Showing Spreadability evaluation of Bhagandharahara Hydrogel

\begin{tabular}{|l|l|l|l|l|l|l|l|}
\hline Parameter & BHG & \multicolumn{5}{l|}{} \\
\hline Time (Day) & 0 & 15 & 30 & 45 & 60 & 75 & 90 \\
\hline Spreadability (g.cm/sec) & 5.00 & 4.62 & 4.10 & 3.40 & 3.40 & 3.40 & 2.88 \\
\hline
\end{tabular}

Table 18: Showing Extrudability evaluation of Bhagandharahara Hydrogel

\begin{tabular}{|l|l|l|l|l|l|l|l|}
\hline Parameter & BHG & \multicolumn{5}{l|}{} \\
\hline Time (Day) & 0 & 15 & 30 & 45 & 60 & 75 & 90 \\
\hline Extrudability (g.cm/sec) & 16.66 & 16.66 & 16.66 & 20.00 & 20.00 & 20.66 & 20.66 \\
\hline
\end{tabular}


Table 19: Showing Irritancy test evaluation of Bhagandharahara Hydrogel

\begin{tabular}{|l|l|l|l|l|l|l|l|}
\hline Parameter & BHG & & \\
\hline Time (Day) & 0 & 15 & 30 & 45 & 60 & 75 & 90 \\
\hline Irritancy test & Non-irritant & Non-irritant & Non-irritant & Non-irritant & Non-irritant & Non-irritant & Non-irritant \\
\hline
\end{tabular}

Table 20: Showing solubility test evaluation of Bhagandharahara Hydrogel

\begin{tabular}{|l|l|}
\hline Solubilizing agent & BHG \\
\hline Methanol & Poorly soluble \\
\hline Ethanol & Poorly soluble \\
\hline Toluene & Not soluble \\
\hline Water & Less soluble \\
\hline Hexane & Not soluble \\
\hline Chloroform & Not soluble \\
\hline
\end{tabular}

\section{Sterility Test}

\section{Total Aerobic Microbial Count}

\section{(a) Plate count:}

For bacteria.

Table 21: showing the table of total aerobic microbial count plate count for bacteria

\begin{tabular}{|l|l|l|}
\hline Sample & No. of colonies in stock solution & No. of colonies in $1^{\text {st }}$ dilution \\
\hline BHG & 5 & 1 \\
\hline
\end{tabular}

\section{For bacteria.}

Table 22: showing the table of total aerobic microbial count plate count for fungi

\begin{tabular}{|l|l|l|}
\hline Sample & No. of colonies in stock solution & No. of colonies in $1^{\text {st }}$ dilution \\
\hline BHG & 5 & 1 \\
\hline
\end{tabular}

(b) Multiple-tube or serial dilution method:

Turbidity or growth in the samples (BHG) test tubes was not observed after incubation period. The positive control showed growth and negative control did not show any growth.

Samples BHG passed the test having less number of organism than the cut-off value set by pharmacopoeia.

\section{Tests for Specified Micro-Organisms}

Table 23: showing the table of tests for specified micro-organisms

\begin{tabular}{|l|l|l|l|}
\hline Bacteria & Agar medium & Sample No. & Observation \\
\hline E. coli & MacConkey & BHG & No colour change was observed and there were no colonies \\
\hline P. aeruginosa & Cetrimide & BHG & No greenish colonies were observed. \\
\hline S. aureus & Mannitol salt & BHG & No change in colour \\
\hline
\end{tabular}

Samples BHG did not show the presence of E. coli, P. aerouginosa and S. aureus.

\section{Melting Point Test}

Table 24: Showing Melting Point test evaluation of Bhagandharahara Hydrogel

\begin{tabular}{|c|c|c|c|c|c|c|c|}
\hline Parameter & BHG & & & & & & \\
\hline Time (Day) & 0 & 15 & 30 & 45 & 60 & 75 & 90 \\
\hline Melting Point $\left({ }^{\circ} \mathrm{C}\right)$ & 108 & 108 & 107 & 107 & 108 & 109 & 110 \\
\hline
\end{tabular}




\section{HPTLC Determination:}

\section{Result:}

1) Standard curcumin: Rf 0.36 AUC: 49777.0

2) BHG: Rf 0.00 AUC: 00

Table 25: Showing HPTLC determination of Bhagandharahara hydrogel Quantification of Curcumin in sample

\begin{tabular}{|l|l|}
\hline Sample & Percentage content of Curcumin \\
\hline BHG & $0 \%$ \\
\hline
\end{tabular}

Stability Test ${ }^{15}$ : (Physical and Chemical Stability) The stability study was performed as per ICH guidelines and formulation were stored under room temperature stability conditions (Temperature $25^{\circ} \mathrm{C} \pm 2{ }^{\circ} \mathrm{C}$,
Humidity $65 \% \pm 5 \% \mathrm{RH}$ ) were carried for a period of 3 months. The physical and chemical analysis was assessed periodically at 15 days interval for a period of 3 months.

Table 26: Showing Physico Organoleptic Stability evaluation of Bhagandharahara hydrogel

\begin{tabular}{|c|c|c|c|c|c|c|}
\hline Sample & Time (Day) & Colour & Odour & Texture & Consistency & Spread ability \\
\hline \multirow[t]{7}{*}{ BHG } & 0 & Light Ochre (colour code 7926) & Characteristics pungent odour & Smooth & Porosity and soft & Easily spreading \\
\hline & 15 & brownish yellow & Characteristics pungent odour & Smooth & Porosity and soft & Easily spreading \\
\hline & 30 & brownish yellow & Characteristics pungent odour & Smooth & Porosity and soft & Easily spreading \\
\hline & 45 & brownish yellow & Characteristics pungent odour & Smooth & Porosity and soft & Easily spreading \\
\hline & 60 & brownish yellow & Characteristics pungent odour & Smooth & Porosity and soft & Easily spreading \\
\hline & 75 & brownish yellow & Characteristics pungent odour & Smooth & Porosity and soft & Easily spreading \\
\hline & 90 & brownish yellow & Characteristics pungent odour & Smooth & Porosity and soft & Easily spreading \\
\hline
\end{tabular}

* The colour of the hydrogel gradually changed over a period of stability studies from Light Ochre to dark brownish yellow *

Table 27: Showing Physico-chemical stability evaluation of Bhagandharahara Hydrogel

\begin{tabular}{|l|l|l|l|l|l|l|l|}
\hline Parameter & BHG & 0 & 15 & 30 & 45 & 60 & 75 \\
\hline Time (Day) & ++ & + & + & + & + & + & + \\
\hline Homogeneity test & 4.90 & 4.63 & 4.46 & 4.41 & 4.41 & 4.29 & 4.29 \\
\hline $\mathrm{P}^{\mathrm{H}}(10 \%$ aqueous extract) & $>30,650$ & $>30,650$ & $>30,650$ & $>30,650$ & $>30,650$ & $>30,650$ & $>30,650$ \\
\hline Viscosity test $(\mathrm{cp})$ & 5.00 & 4.62 & 4.10 & 3.40 & 3.40 & 3.40 & 2.88 \\
\hline Spreadability $(\mathrm{g} . \mathrm{cm} / \mathrm{sec})$ & 16.66 & 16.66 & 16.66 & 20.00 & 20.00 & 20.66 & 20.66 \\
\hline Extrudability $\left(\mathrm{g} / \mathrm{cm}^{2}\right)$ & Non irritant & Non irritant & Non irritant & Non irritant & Non irritant & Non irritant & Non irritant \\
\hline Irritability & 108 & 108 & 107 & 107 & 108 & 109 \\
\hline Melting point $\left({ }^{\circ} \mathrm{C}\right)$ & & & & 110 & + \\
\hline
\end{tabular}

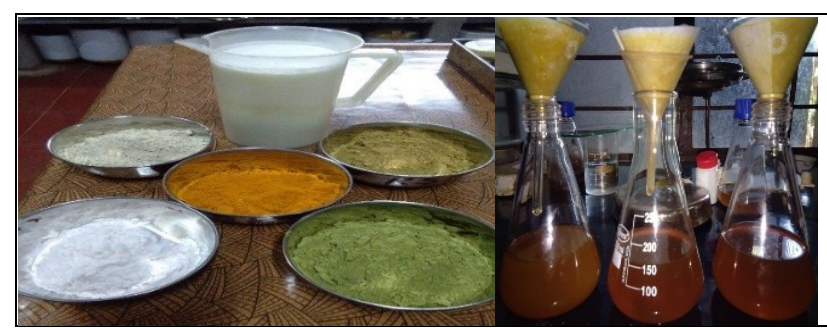

Fig 1: Bhagandharahara Lepa Ingredients
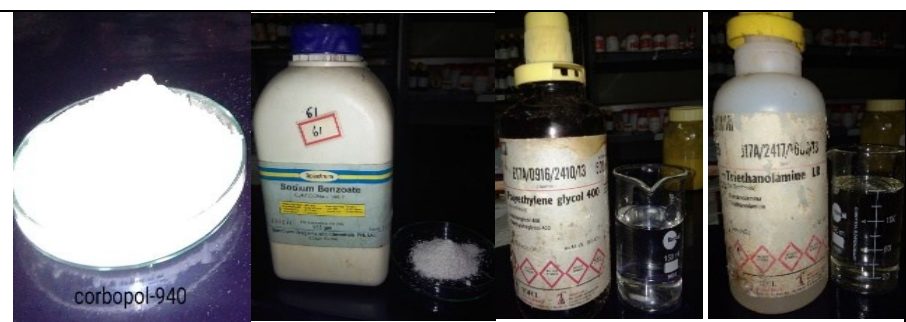

Fig 2: Bhagandharahara Hydrogel Ingredients 


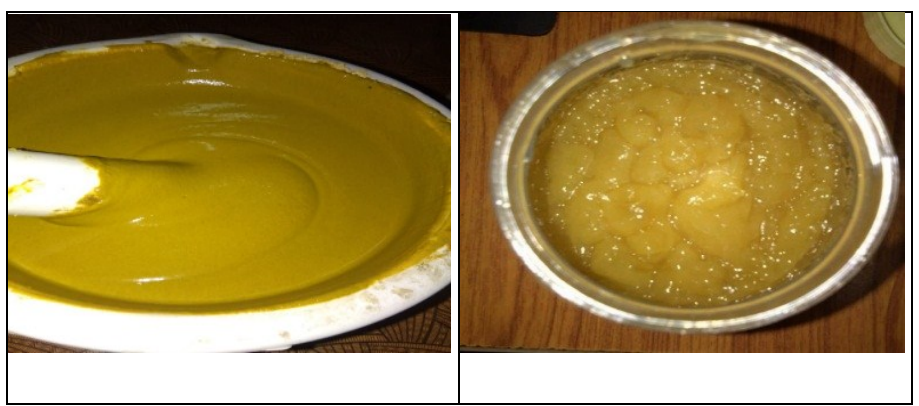

Fig 3: Final product of Bhagandharahara Lepa and Bhagandharahara Hydrogel

\section{DISCUSSION}

Advances in ayurvedic pharmaceutics is just an old ghrita in new bottle the basic principle is same as advice by acharya in Ayurveda classical books, we can change the only way of presentation by using modern technologies.

In ayurvedic clinical practice, practitioner faces difficulties during prescribe Ayurveda dosage form due to its appearance, non-palatable, non-portability. Because of these demerits it's a great challenge in front of Ayurveda pharmaceutics to improve Ayurveda dosage form which will be easily palatable, long selflife simplify to dispense easily portable and good appearance with increasing therapeutic, utility, potency and increase market value. Hence an effort was made to present study is aimed to prepare Bhagandharahara lepa with its modified form as a Hydrogel with pharmaceutical and analytical approach.

In HPTLC Analysis of Bhagandharahara Hydrogel sample, curcumin was not detected. It was found that lepa churna samples is having good content of curcumin and Bhagandharahara Hydrogel having no curcumin content, it may be because Haridra is not dissolved in aqueous extract. Outcome of HPTLC here indicate that hydrogel doesn't contain curcumin due to its insolubility in water but is seen in lepa as the whole drug is to be used.

\section{CONCLUSION}

The drugs used were reviewed and it was found that having several pharmacology actions as anti-inflammatory, Antiallergic, Antifungal, wound healing activity, excellent cleansing property and helpful in treating Bhagandhara disease.
As lepa is having shorter shelf life, it cannot be preserved to longer time, so on this study its modification to hydrogel form facilitates a longer shelf life.

Due to some special features of prepared Bhagandharahara Hydrogel like extrudability, spreadability, viscosity it is easy to apply to the target site.

With regards to the pharmaceutical and dermatological character which includes better stability and skin friendly nature. It looks that modification of lepa into Hydrogel form is a better choice. If preservative is used self-life further can be extended, but pharmacological and clinical intervention are essential to establish the efficacy of Hydrogel as it doesn't contain curcumin.

\section{REFERENCES}

1. Yadavji Trikamji Acharya Susrutha Samhita with Nibandha Sangraha commentary reprint 2008 Varanasi Choukhamba Surabharati Prakashana Chikisthasthana 8/4-page No. -439.

2. Anonymous, Sahasrayogam, translated by Dr. G. Prabhakar Rao (Sanskrit text with English translation \&Prabhakar Vyakhyanam) Choukhamba publication New Delhi. Edition Ist 2016, 12 Chapter. Page No. 337.

3. Anonymous, Sahasrayogam, translated by Dr K. Nishteswar \& Dr. R. Vidyanath, choukhamba Sanskrit series office Varanasi.Edition 3rd 2011, 8th Chapter. Page No. 313.

4. Pandey Gyanendra English, Sanskrit (Materia- Medica Vegetable drugs) Dravyaguna vijnana (Volume 1) edition third - 2005 Choukhamba Krishna Das Academy, Varanasi Page No-737.

5. Pandey Gyanendra English, Sanskrit (Materia- Medica Vegetable drugs) Dravyaguna vijnana (Volume 1) edition third - 2005 Choukhamba Krishna Das Academy, Varanasi Page No-749. 
6. Pandey Gyanendra English, Sanskrit (Materia- Medica Vegetable drugs) Dravyaguna vijnana (Volume 2) edition - reprint 2004 Choukhamba Krishna Das Academy, Varanasi Page No-690.

7. Dr. Ravindra Angadi (A textbook of Bhaisajya kalpana vijnana pharmaceutical science, edition 1st 2009 chapter-22 page No -168-169.

8. Pandey Gyanendra English, Sanskrit (Materia- Medica Vegetable drugs) Dravyaguna vijnana (Volume 1) edition third - 2005 Choukhamba KrishnaDas Academy, Varanasi Page No-214-215.

9. Mishrabhava, Bhavaprakasa (Original text along with commentary \& translation) (including Nigantu portion volume -1 commentary by Bulusu Sitaram 1st Edition 2006 Chukhamba Orientalia 6-chapter page No. 533.

10. Jain NK, Pharmaceutical product development, 6 , CBS publishers and distributors, New Delhi, 2010, page no. 230.

11. Edited by Raymond C Rowe, Handbook of Pharmaceutical Excipients, published by the Pharmaceutical Press 2006, page no.111.

12. Edited by Raymond C Rowe, Handbook of Pharmaceutical Excipients, published by the Pharmaceutical Press 2006, page no.662.

13. Edited by Raymond C Rowe, Handbook of Pharmaceutical Excipients, published by the Pharmaceutical Press 2006, page no.545.

14. Edited by Raymond C Rowe, Handbook of Pharmaceutical Excipients, published by the Pharmaceutical Press 2006, page no. 794 .

15. ICH guidelines stability testing of new drug substances and products, 27th October 1993.

\section{Source of Support: Nil}

\section{Conflict of Interest: None Declared}

How to cite this URL: Hari Anand Tripathi et al: A Comparative Pharmaceutico Analytical Study Of Bhagandharahara Lepa With Its Modified Form As A Hydrogel. International Ayurvedic Medical Journal \{online\} 2020 \{cited May, 2020\} Available from: http://www.iamj.in/posts/images/upload/2309 2318.pdf 